\title{
A High-Throughput Virus-Induced Gene-Silencing Vector for Screening Transcription Factors in Virus-Induced Plant Defense Response in Orchid
}

\author{
Hsiang-Chia Lu, ${ }^{1}$ Ming-Hsien Hsieh, ${ }^{2,3,4}$ Cheng-En Chen, ${ }^{1}$ Hong-Hwa Chen, ${ }^{2,3,5}$ Hsiang-lu Wang, ${ }^{6}$ and \\ Hsin-Hung Yeh ${ }^{1,7}$ \\ ${ }^{1}$ Department of Plant Pathology and Microbiology, National Taiwan University, Taipei 106, Taiwan; ${ }^{2}$ Department of Life Sciences \\ and ${ }^{3}$ Institute of Tropical Plant Sciences, National Cheng Kung University, Tainan 701, Taiwan; ${ }^{4}$ Tainan District Agricultural \\ Research and Extension Station, Council of Agriculture. Tainan 712 Taiwan; ${ }^{5}$ Orchid Research Center, National Cheng Kung \\ University; ${ }^{6}$ Department of Computer Science, National Tsing Hua University, 101, Section 2, Kuang-Fu Road, Hsinchu 30013, \\ Taiwan; ${ }^{7}$ Research Center for Plant Medicine, National Taiwan University
}

Submitted 11 October 2011. Accepted 22 February 2012.

The large number of species and worldwide spread of species of Orchidaceae indicates their successful adaptation to environmental stresses. Thus, orchids provide rich resources to study how plants have evolved to cope with stresses. This report describes our improvement of our previously reported orchid virus-induced gene silencing vector, pCymMV-pro60, with a modified Gateway cloning system which requires only one recombination and can be inoculated by agroinfiltration. We cloned 1,700 DNA fragments, including 187 predicted transcription factors derived from an established expression sequence tag library of orchid, into pCymMV-Gateway. Phalaenopsis aphrodite was inoculated with these vectors that contained DNA fragments of the 187 predicted transcription factors. The viral vector initially triggered the expression of the salicylic acid (SA)related plant defense responses and later induced silencing of the endogenous target transcription factor genes. By monitoring the expression of the SA-related plant defense marker PhaPRl (homolog of PRI), we identified a gene, PhaTF15, involved in the expression of PhaPR1. Knockdown of PhaTF15 by virus-induced gene silencing and by transient delivery of double-stranded RNA (dsRNA) reduced expression of the orchid homolog of the conserved positive defense regulator NPR1, PhaNPR1. Cymbidium mosaic virus also accumulated to high levels with knockdown of PhaTF15 by transient delivery of dsRNA. We demonstrated efficient cloning and screening strategies for high-throughput analysis of orchid and identify a gene, PhaTF 15, involved in regulation of SA-related plant defense.

In this post-genomic era, the number of plant genome and transcriptome sequences derived from whole-genome sequencing and expressed sequence tag (EST) projects has increased exponentially. Especially with the development of next-generation sequencing, the sequencing of the whole genome or

Corresponding author: H.-H. Yeh; E-mail: hyeh@ntu.edu.tw; Fax: +1 886-223636490 .

* The $e$-Xtra logo stands for "electronic extra" and indicates that a supplementary table is published online and that Figures 2, 3, and 4 appear in color online. transcriptome under different conditions can be achieved time and cost effectively. This development can help extend our research and knowledge to important but not easily studied non-model plants. However, the sequences alone do not provide enough information to determine their functions in organisms. Turning this massive amount of sequence information into biological significance functions provides opportunities and challenges for plant biologists.

Various reverse-genetics approaches have been developed for functional validation of genes. One such approach is lossof-function assay by RNA interference (RNAi). RNAi is a well-characterized phenomenon targeting RNA; it is triggered by a double-stranded RNA (dsRNA) mediator to generate small interfering RNA, then causes sequence-specific RNA degradation. The technique has been extensively used to knock down endogenous gene expression for functional validation of genes in various species, including nematodes, Drosophila spp., mammals, and plants (Kuttenkeuler and Boutros 2004; Martin and Caplen 2007; Ohkumo et al. 2008; Small et al. 2007).

In plants, delivery of dsRNA (or hairpin RNA [hpRNA]) can be achieved through plant transformation or transient delivery by particle bombardment, Agrobacterium infiltration, or virus infection (Watson et al. 2005). Although RNAi can last for a long time in stable transgenic lines expressing the dsRNA or hpRNA, the technique is not easily applied to plants with low transformation efficiency and long regeneration time. Even in model plants, obtaining stable transgenic lines still requires extensive tissue culture and is not easily applied to high-throughput functional analysis. dsRNA or hpRNA can be transiently delivered by particle bombardment or Agrobacterium infiltration, with Agrobacterium spp. carrying plasmids harboring the dsRNA or hpRNA; however, the RNAi only lasts for a couple of days and has limited application (Bartlett and Davis 2006; Shang et al. 2007). Alternatively, using a virus as a vector to carry a nonviral sequence and, thus, trigger virus-induced gene silencing (VIGS) in hosts is rapid, easy, and cost effective (Robertson 2004; Wang and Waterhouse 2002). Furthermore, VIGS can be used for functional characterization of genes that might cause lethality in transgenic lines. The effectiveness of high-throughput VIGS was first demonstrated in Potato virus $X$ (PVX) vector (Lu et al. 2003). The roles of 4,992 cDNAs of 
Nicotiana benthamiana in disease resistance were assayed, and heat shock protein 90 (HSP90) was found to play a role in Pto-mediated resistance against Pseudomonas syringae (Lu et al. 2003).

To facilitate high-throughput cloning and analysis, a VIGS vector based on Tobacco rattle virus (TRV) with broad host range and engineered with an improved cloning system, the Gateway-based system, was developed (TRV2) (Liu et al. 2002). The Gateway-based system was originally designed to require two reactions by different enzyme sets: i) the BP reaction for cloning the gene into the Gateway donor vector and ii) the LR reaction for subcloning a gene from the entry vector into destination vectors. This design allows the DNA fragments cloned in the entry vector to be easily recombined to different specially designed destination vectors for expressing proteins in different organisms. To perform VIGS in plants, a small fragment of a target gene ( $>21$ nucleotides [nt]) inserted in a viral vector is enough to trigger RNAi. Thus, to reduce the cost and efforts for high-throughput cloning, the viral vector with recombination sequences derived from the entry vector sequence was adopted for VIGS.

However, a previous report indicated that, with the PDS gene fragment cloned into the TRV vector with Gateway donor vector recombination sequences by BP reaction, the TRV-PDS was not able to induce a photobleaching phenotype in plants (Liu et al. 2002). The authors concluded that, after the BP reaction, the long-directed repeated sequence (100 bp) flanking the inserted PDS sequences in the vector may lead to deletion of inserted sequences with infection and fail to induce the target gene silencing (Liu et al. 2002). Thus, the whole Gateway cloning system, which requires the silencing fragment first cloned to the Gateway donor by the BP reaction and then the LR reaction (reducing the flanking repeat to $25 \mathrm{bp}$ ) to TRV2, is engineered with the recombination sites derived from the Gateway destination vectors. Because TRV2 requires multiple steps to clone the silencing fragment into the vector and each step requires expensive enzymes for cloning, a ligation-independent cloning (LIC) system of TRV VIGS vector was developed (Dong et al. 2007).

Orchidaceae is a widespread family of monocots of an estimated more than 35,000 species, one of the largest families of the flowering plants (Dressler 1993). The diverse large species and worldwide spread of orchids indicates their successful adaptation to abiotic and biotic stresses. Thus, versatile orchids provide rich resources to study the strategy, mechanism, and evolution of plants in coping with stresses. However, our knowledge about orchids is still limited because of the lack of an easy genetic analysis system, difficulties in regeneration, and the slow growth of orchids.

Biotrophic pathogens, including viruses, usually trigger a salicylic acid (SA)-related plant defense response (McDowell and Dangl 2000). On infection, the virus induces the accumulation of SA in both inoculated and systematic leaves of plants and triggers the expression of several pathogenesis-related (PR) genes (Durrant and Dong 2004; Loake and Grant 2007; Sticher et al. 1997; Van Loon and Van Kammen 1970; Van Loon and Van Strien 1999). The accumulation of SA also triggers systemic acquired resistance (SAR) in plants, which is effective against a broad spectrum of pathogens distal from the infection site (Durrant and Dong 2004; Loake and Grant 2007; Sticher et al. 1997). Genes involved in the SA-related plant defense response have been extensively studied by genetic screens, and several genes involved in the response have been identified, including the conserved central positive regulator NPR1 (Durrant and Dong 2004; Pieterse and Van Loon 2004). Considerable efforts have focused on how NPR1 transduces the SA signal to activate (PR) gene expression (Grant and
Lamb 2006). NPR1 is controlled at the protein level. When cells are in a noninduced state, NPR1 is present as an oligomer and, during the induction of SAR, the cellular reduction potential changes and results in reduction of NPRI to a monomeric form (Mou et al. 2003). Monomeric NPRI accumulates in the nucleus and regulates the expression of defense-related genes, including PR genes, through action on TGA and WRKY types of transcription factors (TF) (Durrant and Dong 2004; Grant and Lamb 2006). Current data also suggest that the basal and induced expression of NPRI is controlled by WRKY-type TF (Yu et al. 2001). However, this TF has not been identified.

We previously developed a VIGS vector (pCymMV-pro60) and strategies to analyze the function of genes in orchids ( $\mathrm{Lu}$ et al. 2007). Recently, we have identified NPRI and PR-1 homologs from Phalaenopsis orchids. Similar to dicots, the orchid PRI homolog (PhaPRl) is regulated by the orchid NPRl (PhaNPRl). In this report, we describe our improvement of the pCymMV-pro60 vector with better inoculation and cloning strategies. This new vector, pCymMV-Gateway, is suitable for high-throughput VIGS analysis in orchids. With this high-throughput VIGS system, we could study the plant defense regulatory mechanism of orchids and developed a strategy to screen TF involved in virus-induced-related plant defense response. Our high-throughput cloning and analysis strategies allowed us to identify a gene, PhaTF15, upstream of the important plant defense regulator PhaNPRl, from genetically not-easily-manipulated Phalaenopsis orchids. These cloning and screening strategies may be applied to other plants (especially non-model plants) for high-throughput analysis, and the gene PhaTF 15, involved in the regulation of PhaPRl and PhaNRPl, may further help resolve the SA-related plant-defense regulatory mechanism.

\section{RESULTS}

\section{Development of a high-throughput}

Cymbidium mosaic virus (CymMV)-based VIGS vector.

Although our previous VIGS vector, pCymMV-pro60 (Lu et al. 2007), could be used for functional gene validation in orchids, the use of the vector required in vitro transcription to obtain infectious transcripts is time consuming and cost ineffective. To be more efficient for high-throughput VIGS analysis of orchids, we inserted the CymMV vector sequence of pCymMV-pro60 into the binary vector, pCAMBIA 1301, under a double $35 \mathrm{~S}$ promoter (Fig. 1), which allowed the infectious viral RNA to be generated in plants via the double $35 \mathrm{~S}$ promoter by infiltration of Agrobacterium spp. containing the vector.

In addition, we engineered a modified Gateway cloning system in the cloning site of CymMV to construct pCymMVGateway (Fig. 1A). We inserted only the $\lambda$ phage core recombination sequences (Hartley et al. 2000) of the Gateway donor vector (Fig. 1A). This cloning strategy required only one recombination reaction (BP reaction) and allowed the repeated sequences flanking the foreign sequences to be reduced from 100 to $25 \mathrm{bp}$ after BP reaction, which would increase the stability of the foreign DNA inserted in the vector. We used this strategy to clone 1,700 orchid uni-genes, including 187 predicted TF derived from an established EST library (Tsai et al. 2006). The recombination efficiency was reduced, as observed by decreased number of colonies; however, we could still obtain 30 to 50 colonies with 200-ng plasmids used for transformation. For the 1,700 pCymMV-Gateway EST, approximately 94\% of clones contained the expected size of inserts from the first selected Escherichia coli colony in each plate. Therefore, the modified Gateway system was still efficient in cloning foreign DNA. 
To test the infection rate and efficacy in inducing gene silencing with pCymMV-Gateway, we used pCymMV-Gateway containing a 150-bp stretch derived from the $3^{\prime}$ terminus of a Phalaenopsis B-class MADS box gene (PeMADS6), pCymMVGateway-PeMADS6(150), to inoculate Phalaenopsis aphrodite. This clone can specifically knock down PeMADS6 but not other B-class MADS box genes (Lu et al. 2007). The infection rate of pCymMV-Gateway-PeMADS6 was slightly lower than that of the previously reported pCymMV-pro60-PeMADS6, (Lu et al. 2007) but still reached approximately 67\%. We randomly selected three plants to calculate the relative reduction of PeMADS6 in orchids with successful infection by real-time reverse-transcriptase polymerase chain reaction (RT-PCR). The gene silencing efficacy was similar between pCymMVpro60-PeMADS6(150) (55 to 63\% reduction) and pCymMVGateway-PeMADS6 (59 to $65 \%$ reduction). Because we previously found that pCymMV-pro60-PeMADS6(150) did not induce an observable phenotype in orchids (Lu et al. 2007), we inserted a 500-bp conserved region of MADS box gene from PeMADS6 into pCymMV-Gateway (pCymMV-GatewayPeMADS6(500)). As previously reported (Lu et al. 2007), both viral vectors (pCymMV-pro60 and pCymMV-GatewayPeMADS6) containing this region could induce a leaf-like structure in infected orchids (Fig. 1B), and more than $90 \%$ of orchids (at least 30 orchids inoculated for each vector) showed the leaf-like structure.

\section{Identification of PhaTF 15 involved}

in virus-induced plant resistance

by high-throughput pCymMV-Gateway VIGS analysis.

We selected pCymMV-Gateway containing the 187 DNA fragments of predicted TF for screening putative TF involved in virus resistance. Viruses can trigger a host immune response and induce gene silencing. Thus, if a viral vector carries partial sequences of a TF, the viral vector first triggers the plant defense response and then induces gene silencing and also silences the endogenous gene expression of the TF (Fig. 2). Therefore, if the TF is involved in the plant defense response, monitoring the marker gene of the plant defense response will allow us to screen TF involved in the plant defense response (Fig. 2). Previous reports indicated that $P R-1$ is a good marker for monitoring the SA-related plant defense response (Uknes et al. 1992). We previously cloned the entire sequence of the orchid PR-1 homolog, PhaPRl (accession number JN630802), and the induction of PhaPRl with SA treatment and CymMV infection was previously demonstrated. By this approach, our screening identified PhaTF15 involved in the transcription of PhaPRl (Fig. 3A).

\section{Sequence analysis of PhaTF15.}

Sequence analysis revealed that PhaTF15 contained a conserved RING-finger domain (Fig. 3B and C). Phylogenetic analysis with the homolog of PhaTF15 revealed three groups and the PhaTF15 homolog genes derived from monocotyledons in the same group (Fig. 4).

\section{PhaTF15 is involved in the regulation of PhaPR-1, PhaNPR-1, and virus accumulation.}

Our initial analysis of the whole cDNA sequences derived from the established EST inserted into pCymMV-Gateway increased the possibility of an off-target effect. To more specifically analyze the role of PhaTF15 in SA-related plant defense, we inserted 21-nt inverted DNA of PhaTF15 into pCymMVGateway (pCymMV-Gateway-TF15-21(IR)) for VIGS analysis. We observed silencing of PhaTF15 in four of six pCymMV-Gateway-TF15-21(IR)-inoculated plants and reduced

\section{A pCymMV-Gateway}

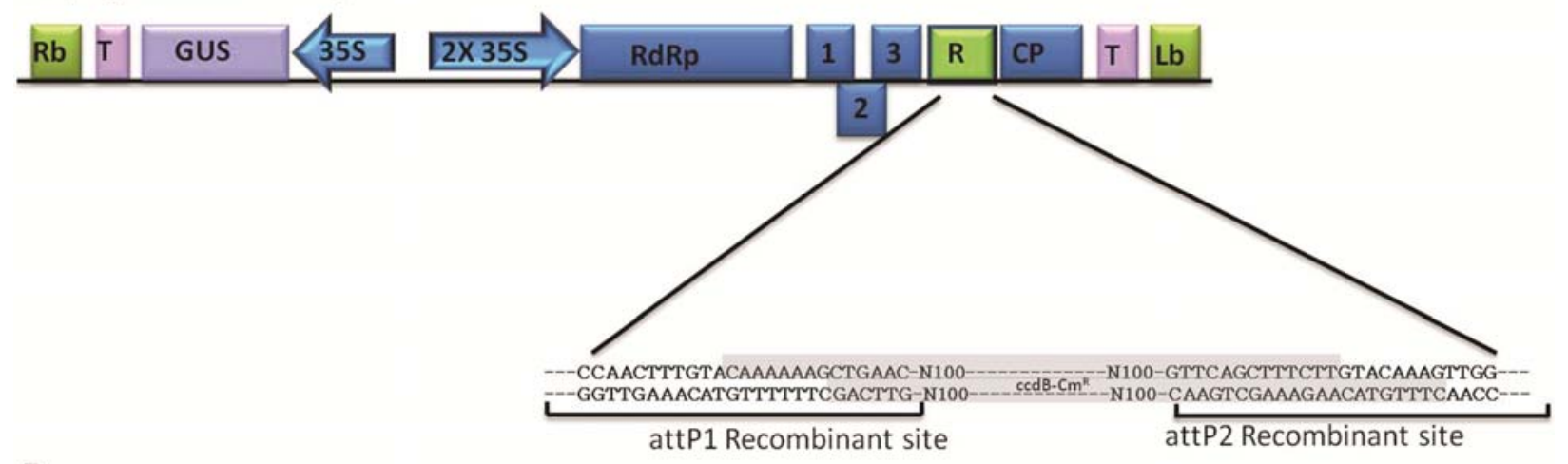

B

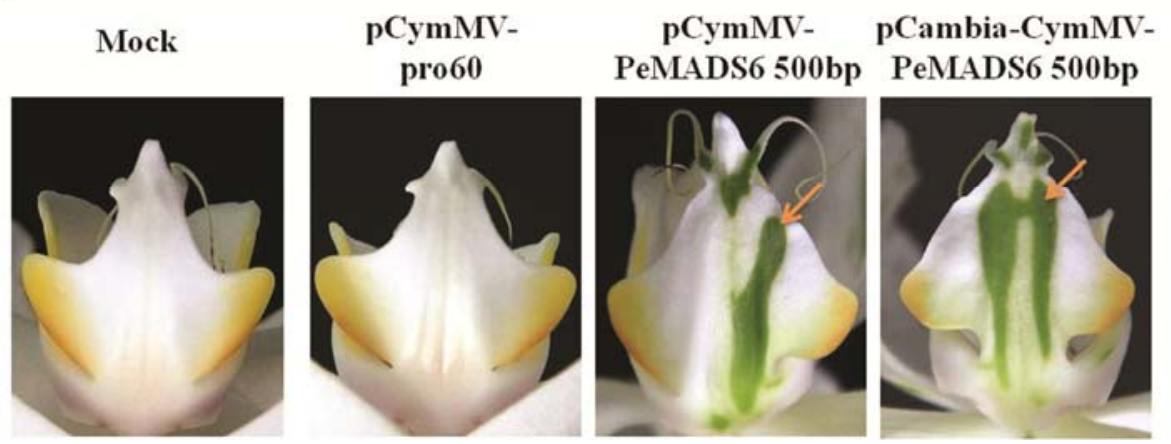

Fig. 1. Schematic representation of pCymMV-Gateway infectious clone and phenotype in MADS box gene-silenced plants. A, Blue rectangles represent open reading frames encoded by Cymbidium mosaic virus (CymMV) genomic RNA. RNA-dependent RNA polymerase (RdRp); triple gene block 1, 2, and 3 ; and coat protein (CP) are indicated. The $35 \mathrm{~S}$ promoter is immediately adjacent to the CymMV sequence at pCymMV-Gateway. The BP recombinant reaction sequence on pCymMV-Gateway is shown. Recombinant sequences are labeled by gray blocks. B, Plants were inoculated with buffer (Mock), pCymMV-pro60, pCymMV-pro60-PeMADS6(500), and pCymMV-Gateway-PeMADS6(500). Arrows point to greenish patches on the lip. 
mRNA levels of PhaPRl and PhaNPRl in only these four silenced plants (Fig. 5A, lanes 1, 2, 4, and 6).

To gain more evidence to support PhaTF15 involved in PhaPRl and PhaNPRl induction, we performed another transient knockdown analysis. We delivered PhaTF15 (21 nt)
hpRNA into SA-treated orchids by infiltration of Agrobacterium spp. carrying pB7GWIWG2 (Karimi et al. 2002) inserted with two 21-nt PhaTF15 DNA fragments (pB7GWIWG2PhaTF15-hpRNA1 and pB7GWIWG2-PhaTF15-hpRNA2). The hpRNAs would be transcribed by a $35 \mathrm{~S}$ promoter and in-
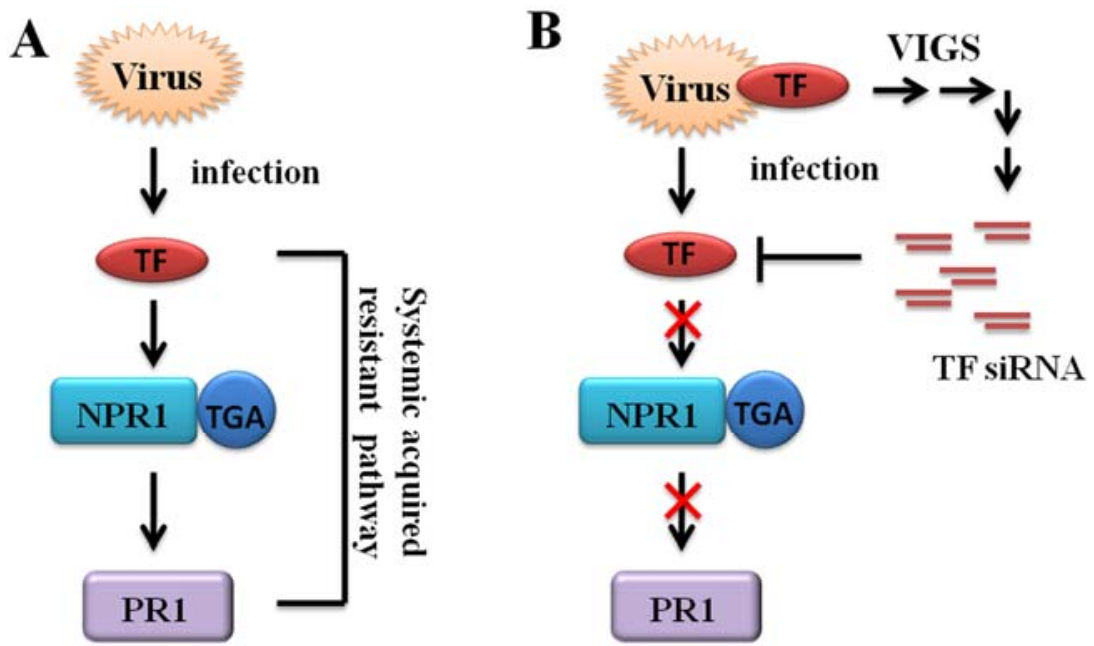

Fig. 2. Strategy of analyzing systemic acquired resistance (SAR)-related transcription factors (TF) in Phalaenopsis orchid. A, Cymbidium mosaic virus (CymMV)-infected plants may trigger salicylic acid (SA)-related plant defense, and the defense response will induce the expression of NPR1 or PR1 genes. $\mathbf{B}$, If the viral vector carries partial sequences of a TF, the viral vector first triggers the plant defense response and then induces gene silencing and silences the endogenous gene expression of the TF. If the TF is involved in SA-related plant defense, the expression of NPR1 or PR1 genes will be blocked. VIGS = virus-induced gene silencing.

A

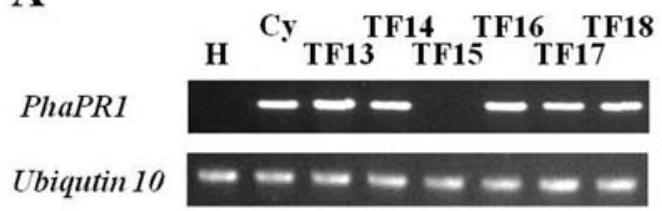

B

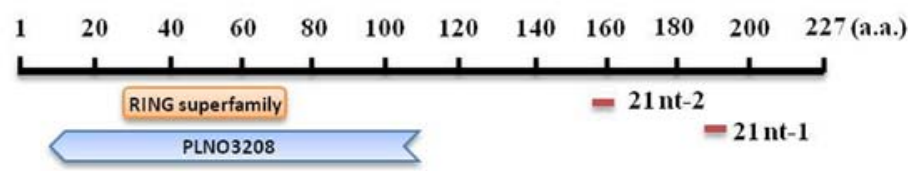

C

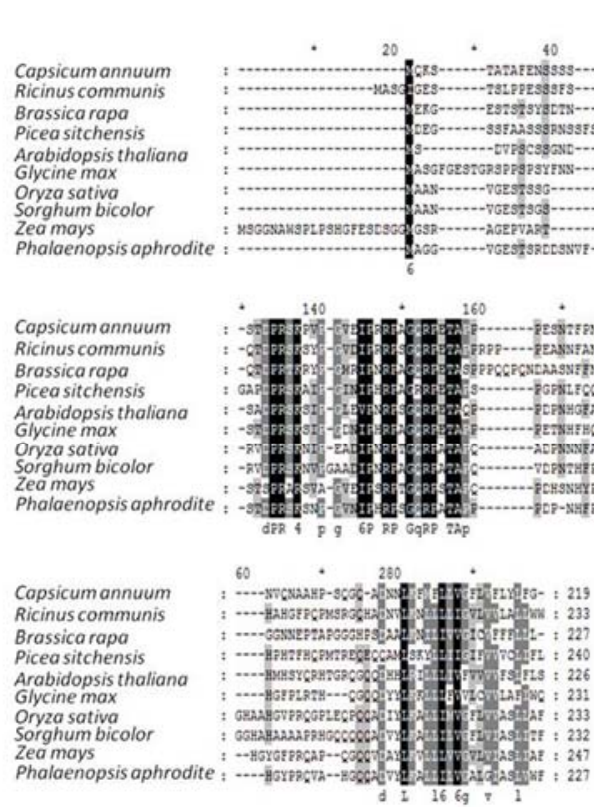

Fig. 3. Semi-quantitative reverse-transcriptase polymerase chain reaction analysis of pathogenesis-related 1 ( $P R 1)$ and sequence analysis of $P h a T F 15$. A, PRI and Ubiquitin gene expression in leaves of healthy plants $(\mathrm{H})$, pCymMV-infected plants $(\mathrm{Cy})$, and plants with various transcription factor $(\mathrm{TF})$ silencing (TF 13-18). At least 187 plants were analyzed, and only part of the results are shown. B, Map of the RING-finger domain and 21-nucleotide (nt) position in PhaTF15. C, Sequence alignment of PhaTF15 with RING-finger proteins from Zea mays (accession number ACR37583), Arabidopsis thaliana (NP_564078), Brassica rapa (ADK63399), Capsicum annuum (ACN63363), Oryza sativa (NP_001050892), Picea sitchensis (ABR17325), Sorghum bicolor (_002442611), Ricinus communis (XP_002533271), and Glycine max (ACU19464). The conserved RING-finger domain is boxed. The sequence was aligned by use of ClustalW 1.8. Gaps introduced to maximize alignment are indicated by dashes (-). 


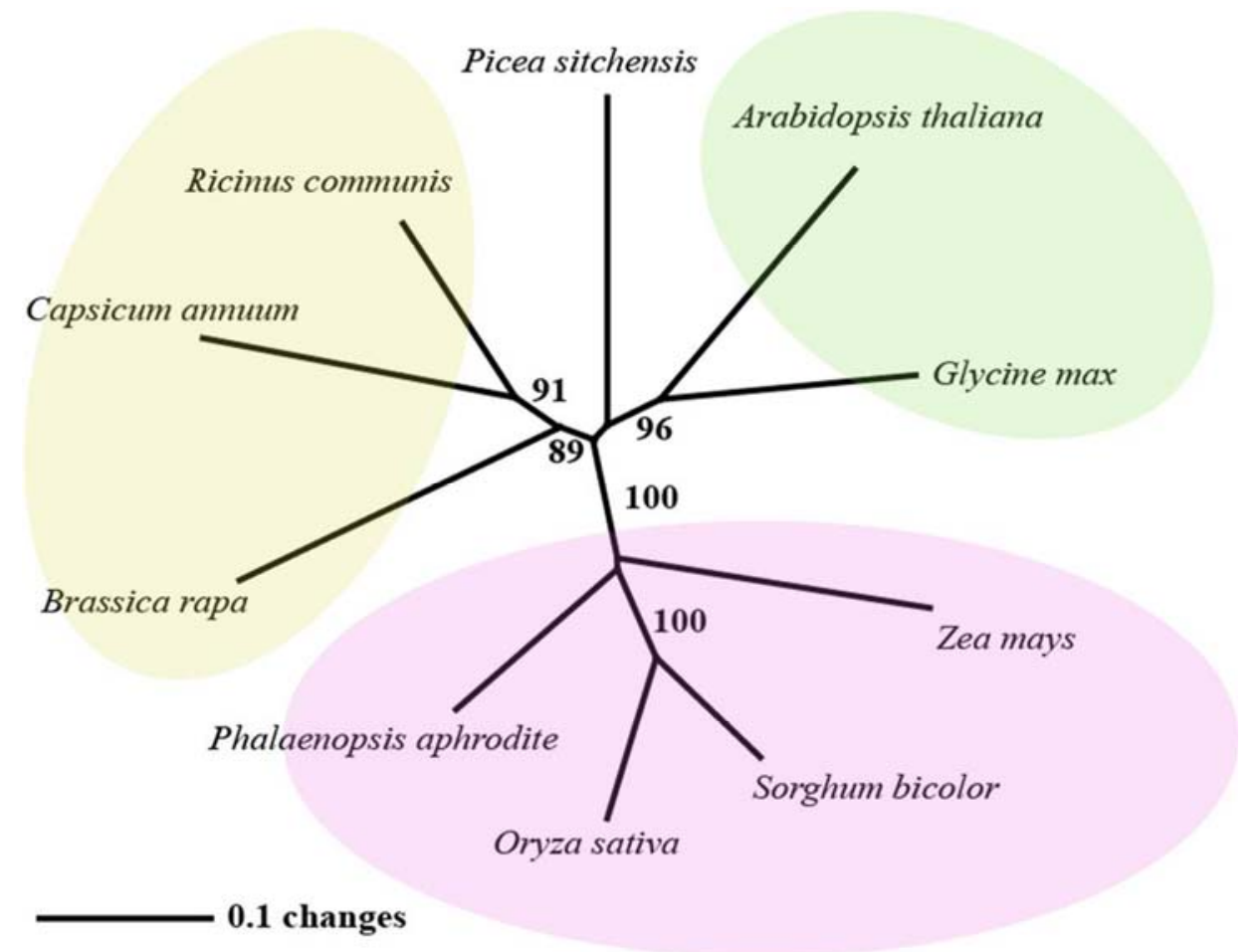

Fig. 4. Phylogram of the neighbor-joining tree based on amino acid sequences forming RING-finger proteins. Branches show the bootstrap supports of the neighbor-joining method.

A

pCymMV-Gateway-TF15-21(IR)

\begin{tabular}{|c|c|c|c|c|c|c|c|c|}
\hline & $\mathrm{H}$ & $\mathrm{Cy}$ & 1 & 2 & 3 & 4 & 5 & 6 \\
\hline PhaPR1 & & $=$ & $c^{2}$ & $\mathrm{ca}$ & 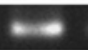 & 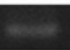 & - & \\
\hline PhaNPR1 & & $=$ & $=$ & $=$ & - & - & - & - \\
\hline PhaTF15 & 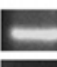 & E & 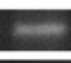 & $=$ & 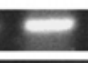 & - & $=$ & \\
\hline CymMV & & - & - & - & & $=$ & & 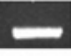 \\
\hline
\end{tabular}

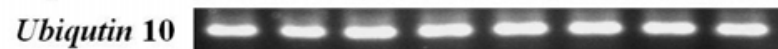

B

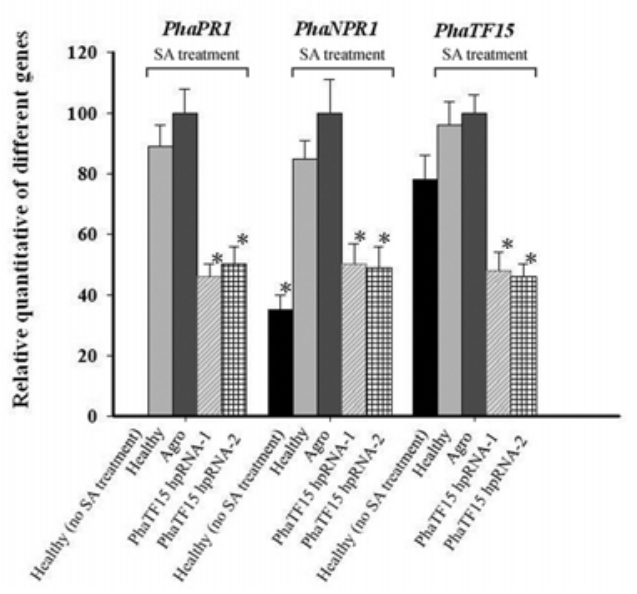

C

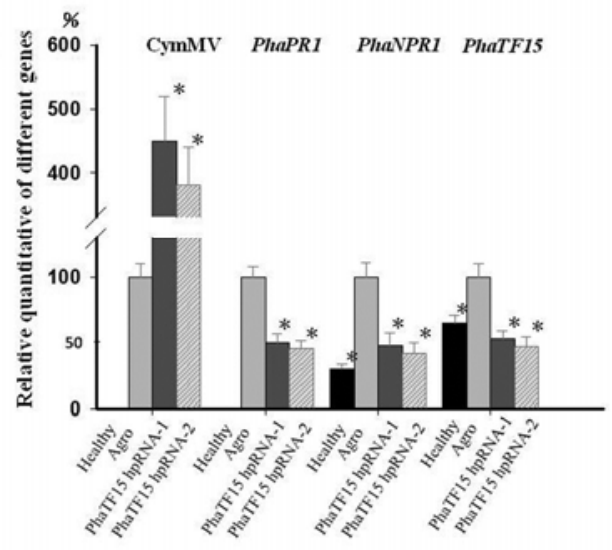

Fig. 5. Quantification of PhaPR1, PhaNPR1, and Cymbidium mosaic virus (CymMV). A, Semi-quantification was performed to measure the expression of PhaPR1, PhaNPR1, Ubiquitin, and PhaTF15 in leaves of healthy plants (H) or plants inoculated with pCymMV-Gateway (Cy) or pCymMV-Gateway-TF15-(21 IR) (lanes 1 to 6). B, Real-time reverse-transcriptase polymerase chain reaction (RT-PCR) was used to measure the relative quantification of PhaPR1, PhaNPR1, and PhaTF15 in leaves of healthy plants $(\mathrm{H})$ or plants infiltrated with Agrobacterium spp. carrying pB7GWIW2 (Agro), pB7GWIW2-PhaTF15-hpRNA-1 (PhaTF15-hpRNA-1), or pB7GWIW2-PhaTF15-hpRNA-2 (PhaTF15-hpRNA-1). C, Real-time RT-PCR was used to measure the relative level of CymMV and PhaPR1, PhaNPR1, and PhaTF15 in leaves of healthy plants (H) or CymMV (wild-type)-infected plants infiltrated with Agrobacterium spp. carrying pB7GWIW2 (Agro) or pB7GWIW2-PhaTF15-hpRNA (PhaTF15-hpRNA). Plant RNA was extracted and analyzed 4 days after agroinfiltration. For B and C, plants inoculated with Agrobacterium spp. carrying pB7GWIW2 (Agro) were set to $100 \%$ for relative quantification. Error bars represent standard deviation. Data were analyzed by Dunnett's $t$ test. An asterisk (*) indicates $P<0.05$ compared with the Agro inoculation. Plants treated with salicylic acids (SA) are indicated. 
duce endogenous PhaTF 15 gene silencing. The mRNA expression of PhaPRl and PhaNPRl was reduced in SA-treated PhaTF15 with transient knockdown of PhaTF15 with both constructs (Fig. 5B).

To determine whether PhaTF15 is involved in plant defense against virus infection, we infiltrated CymMV-infected plants with Agrobacterium spp. carrying pB7GWIWG2-PhaTF15hpRNA1 and pB7GWIWG2-PhaTF15-hpRNA2). Virus accumulation was increased in plants with infiltration of both constructs with PhaTF15 transient knockdown (Fig. 5C).

\section{Subcellular localization of PhaTF15.}

Because PhaTF15 was predicted to be a putative TF, we analyzed the subcellular localization of PhaTF15 protein. We fused green fluorescent protein (GFP) to the $\mathrm{N}$ terminus (GFPPhaTF15) or C terminus (PhaTF15-GFP) of PhaTF15 for transient expression in protoplasts of $P$. aphrodite var. formosa. All PhaTF15-GFP and approximately 50\% of GFP-PhaTF15 fusion protein localized in the nucleus of $P$. aphrodite var. formosa protoplasts (Fig. 6) but control GFP proteins localized throughout the cytoplasm and nucleus. Therefore, PhaTF15 can move to the nucleus.

\section{DISCUSSION}

In this report, we describe our improvement of our previously developed pCymMV-pro60 vector for high-throughput VIGS analysis. We constructed the new vector, pCymMVGateway, within a binary vector (pCambia1301) to generate viable viral RNA de novo by a $35 \mathrm{~S}$ promoter by infiltration of plants with Agrobacterium spp. carrying the vector. In addition, we also report a strategy to facilitate the high-throughput cloning. This strategy achieves high cloning efficiency and maintains the vector stability but reduces the steps needed with regular Gateway cloning. We verified the effectiveness of this vector by developing a high-throughput screening strategy to analyze genes involved in the virus-induced plant defense response. We identified a gene, PhaTF15, positively regulating the orchid homolog (PhaNPRl) of the master plant-defense regulatory gene $N P R 1$.

One of the concerns in applying VIGS for validating gene function is that the analysis is under stress caused by the virus infection, although this problem can be alleviated by setting appropriate controls. We used high-throughput VIGS to screen genes involved in the plant defense response to viral infection. The screened targets are normally under virus infection, and the viral vector is the inducer of plant defense and the trigger of RNAi; thus, VIGS is suitable for such screening.

Much of the SA-related plant defense mechanism has been analyzed in dicots; however, many key players involved in SA-related plant defense, including $N P R l$, can be found in monocots. Recent evidence suggests that SA also induces the plant defense response in monocots but the mechanism is less understood (Vlot et al. 2009). Similar to studies in Arabidopsis, our previous work identified PhaNPRl and PhaPRl, both induced by virus infection or SA treatment; and PhaNPRl was also upstream of PhaPRl (Figs. 5; data not shown). Therefore, Phalaenopsis orchids may adopt a central plant-defense regulatory machinery similar to that of dicots.

Interestingly, we identified PhaTF15 involved in the regulation of the SA-related plant defense response, and our data suggest that PhaTF15 is upstream of PhaPRl and PhaNPRl. However, in contrast to previous suggestions that NPRl may be regulated by a $W R K Y$-type TF (Yu et al. 2001), the TF we identified, PhaTF15, that may be involved in regulation of PhaNPRl is a non-WRKY-type gene. The upstream regulation of SA-related plant defense may differ between dicots and monocots. Our phylogenetic analysis revealed the monocot homologs of PhaTF15 grouped in the same clade. In addition, PhaTF 15 may be indirectly involved in regulating PhaNPRl. PhaTF 15 belongs to a C3HC4-type RING-finger protein family. Proteins within this family may have roles as TF, and our protein localization data supported the idea that PhaTF15 may function as a TF. However, proteins within this family have been reported to bind several substances, including DNA, RNA, protein, and lipids, and be involved in diverse biological functions, including transcription, signal transduction, and stress (Ma et al. 2009; Zeba et al. 2009). Thus, PhaTF15 may be involved in different regulations of the plant defense response in addition to its role as a TF. In addition, because PhaTF15 expressed at similar levels in both healthy and CymMV-infected plants, basal levels of PhaTF 15 may be required for the induction of PhaNPRl, or PhaTF15 is regulated at the protein level like Arabidopsis NPRI (Mou et al. 2003).

We found several homologs of PhaTF15 (Fig. 4) that share high amino acid identity (54 to $71 \%$ ); however, the biological functions of most of these homologs have not been identified. Within these homologous genes, CaRZFP1, found in Capsicum annuum, was induced in response to high or low temperature, drought, and high salinity (Zeba et al. 2009). Whether CaRZFP1 also has a role in response to biotic stress like PhaTF15 remains to be tested.

\section{MATERIALS AND METHODS}

Plants growth conditions and chemical application.

$P$. aphrodite var. formosa, a commercial orchid variety, was purchased from the Taiwan Sugar Research Institute (Tainan, Taiwan). Plants were kept in an insect-proof and thermal-controlled greenhouse with a $12-\mathrm{h}$ photoperiod $\left(200 \mu \mathrm{mol} \mathrm{m} \mathrm{m}^{-2} \mathrm{~s}^{-2}\right)$ at 25 and $25^{\circ} \mathrm{C}$ (day and night, respectively). To ensure that plants were free of virus infection, they underwent RT-PCR with the primer pairs ORSV-CP-F/ORSV-CP-R and CymMV$\mathrm{CP}-\mathrm{F} / \mathrm{CymMV}-\mathrm{CP}-\mathrm{R}$ targeting the coat proteins of two prevalent orchid viruses, Odontoglossum ringspot virus (ORSV) and CymMV (Lu et al. 2007). For analysis of marker genes of the plant defense response, we used SA (10 mM in water) (Sigma, St. Louis) to spray plants. Control plants were sprayed with water.

\section{Construction of pCymMV-Gateway vector.}

The pCymMV-Gateway vector was derived from pCymMVpro60, as described (Lu et al. 2007). pCymMV-pro60 was used as the template, and the primer pairs CymMV F1 and CymMV R1846 (Table 1) were used for PCR to amplify the CymMV fragment. The amplified fragments were ligated to StuI-digested pCASS2 to construct pCass2-CymMV1846. pCass2-CymMV1846 was digested with SphI and EcoRI, then ligated to SphIand EcoRI-predigested pCambia1301 to construct pCambiaCymMV1846. pCymMV-pro60 was digested with MluI and EcoRI, then ligated to MluI- and EcoRI-predigested pCambiaCymMV1846 to construct pCambia-CymMV-pro60. pDONR221 (Invitrogen, Carlsbad, CA, U.S.A.) was used as the template DNA, and the primer pair attP1 and attP2 (Table 1) was used for PCR to amplify the attp recombination sequence and $c c d B$ gene. The amplified fragments were ligated to SmaIdigested pCambia-CymMV-pro60 to construct pCymMVGateway.

\section{Construction of TF into pCymMV-Gateway vector.}

The 187 putative TF (Supplementary Table S1) were cloned in pBluecript vector (Agilent Technologies, Santa Clara, CA, U.S.A.). The clones were used as templates, and TF-attB1-F and TF-attB2-R (Table 1) were used as the primer pair to am- 
plify $187 \mathrm{TF}$ by PCR. The PCR program was $94^{\circ} \mathrm{C}$ for $2 \mathrm{~min}$; 30 cycles of $94^{\circ} \mathrm{C}$ for $30 \mathrm{~s}, 55^{\circ} \mathrm{C}$ for $30 \mathrm{~s}$, and $72^{\circ} \mathrm{C}$ for $90 \mathrm{~s}$; then $72^{\circ} \mathrm{C}$ for $7 \mathrm{~min}$. The amplified fragments were cloned into the pCambia-CymMV vector with use of the BP Clonase II enzyme mix (Invitrogen). For further cloning, other DNA fragments to pCambia-CymMV and the attB1 and attB2 sequences (Table 1) were added to the $5^{\prime}$ end of gene-specific sequences.

\section{Construction of PhaTF15 tagged with GFP.}

The fragment of PhaTF15 was amplified by PCR with plant RNA used as a template and the primer pair PhaTF15ORFF and PhaTF15ORFR (Table 1). The PhaTF15 fragment was cloned into the pCR/GW/TOPO Gateway Entry Vector (Invitrogen) following the manufacturer's recommendations. The pCR/GW/TOPO-PhaTF15 construct was sequenced to confirm the accuracy of the cloned fragments; then, LR Clonase II enzyme (Invitrogen) was used to transfer the cloned fragments into p2FGW7 and p2GWF7, thus generating p2FGW7-GFPPhaTF15 and p2GWF7-PhaTF15-GFP, respectively.

\section{Construction of pB7GWIWG2-PhaTF15-hpRNA.}

The primer pairs PhaTF15-hpRNA1(F) and PhaTF15hpRNA1(R) and PhaTF15-hpRNA2(F) and PhaTF15hpRNA2(R) (Table 1) were used to generate PhaTF15hpRNA-1 and PhaTF15-hpRNA-2 dsDNA. In total, $2 \mu \mathrm{l}$ of each primer $(15 \mu \mathrm{g} / \mu \mathrm{l})$ was mixed with its complementary primer at $72^{\circ} \mathrm{C}$ for $10 \mathrm{~min}$, then transferred to $25^{\circ} \mathrm{C}$ for another
10 min. The double-stranded fragment was cloned into the Gateway Entry Vector pDONR221 (Invitrogen) following the manufacturer's recommendations to generate pDONR221PhaTF15-hpRNA-1 and pDONR221-PhaTF15-hpRNA-2. The pDONR221-dsDNA construct was sequenced to confirm the accuracy of the cloned fragments; then, LR Clonase II enzyme (Invitrogen) was used to transfer the PhaTF15-hpRNA-1 and PhaTF15-hpRNA-2 fragments into pB7GWIWG2 (Karimi et al. 2002) to generate pB7GWIWG2-PhaTF15-hpRNA-1 and pB7GWIWG2-PhaTF15-hpRNA-2, respectively.

\section{Construction of pCymMV-GATEWAY-TF15-21IR.}

pB7GWIWG2-PhaTF15-hpRNA-1 was used as template and PhaTF15-hpRNA1 F and PhaTF15-21IR R were used as the primer pair (Table 1) to amplify the PhaTF15 21-nt inverted repeat sequence by PCR. The PCR program was $94^{\circ} \mathrm{C}$ for $2 \mathrm{~min}$; 30 cycles of $94^{\circ} \mathrm{C}$ for $30 \mathrm{~s}, 55^{\circ} \mathrm{C}$ for $30 \mathrm{~s}$, and $72^{\circ} \mathrm{C}$ for $90 \mathrm{~s}$; and then $72^{\circ} \mathrm{C}$ for $7 \mathrm{~min}$. The amplified fragment was gel purified by use of a gel extraction kit (Viogene Biotek Corp., New Taipei City, Taiwan) and was cloned into the pCambia-CymMV vector by use of BP Clonase II enzyme mix (Invitrogen).

\section{Agroinfiltration.}

pCambia-CymMV-Gateway, pB7GWIWG2, and their derivatives were transformed into Agrobacterium tumefaciens LBA4404 by electroporation. The A. tumefaciens strains were cultured in $2 \mathrm{ml}$ of yeast-extract buffer (YEB) medium (beef extract at $5 \mathrm{~g} / \mathrm{liter}$, yeast extract at $1 \mathrm{~g} / \mathrm{liter}$, peptone at $5 \mathrm{~g} / \mathrm{liter}$,
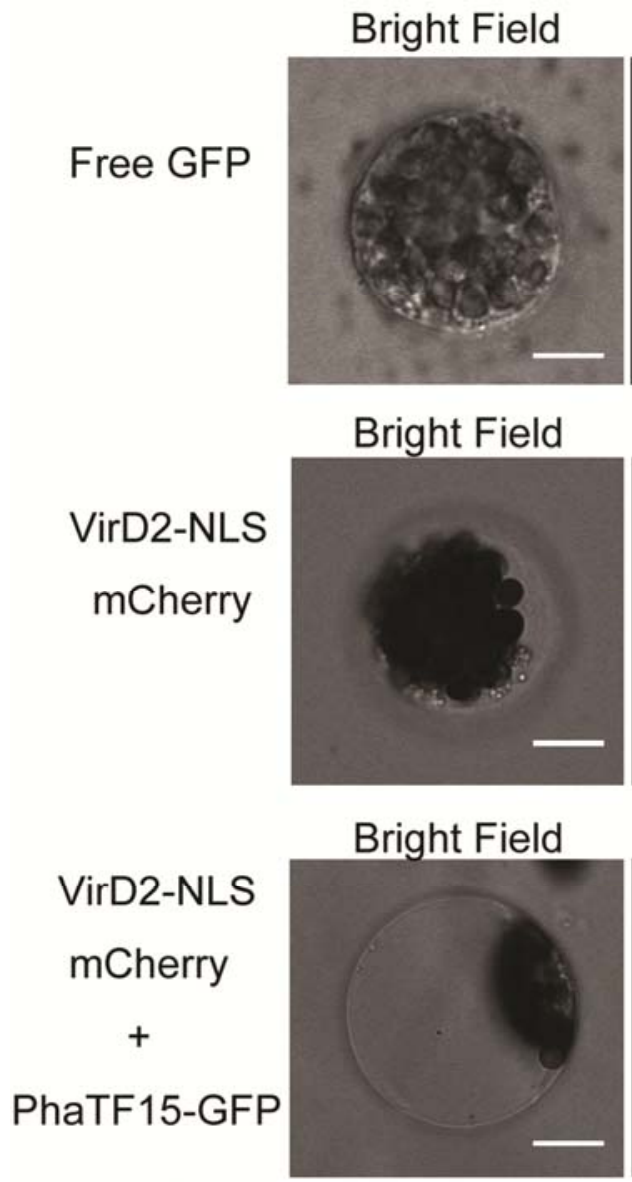
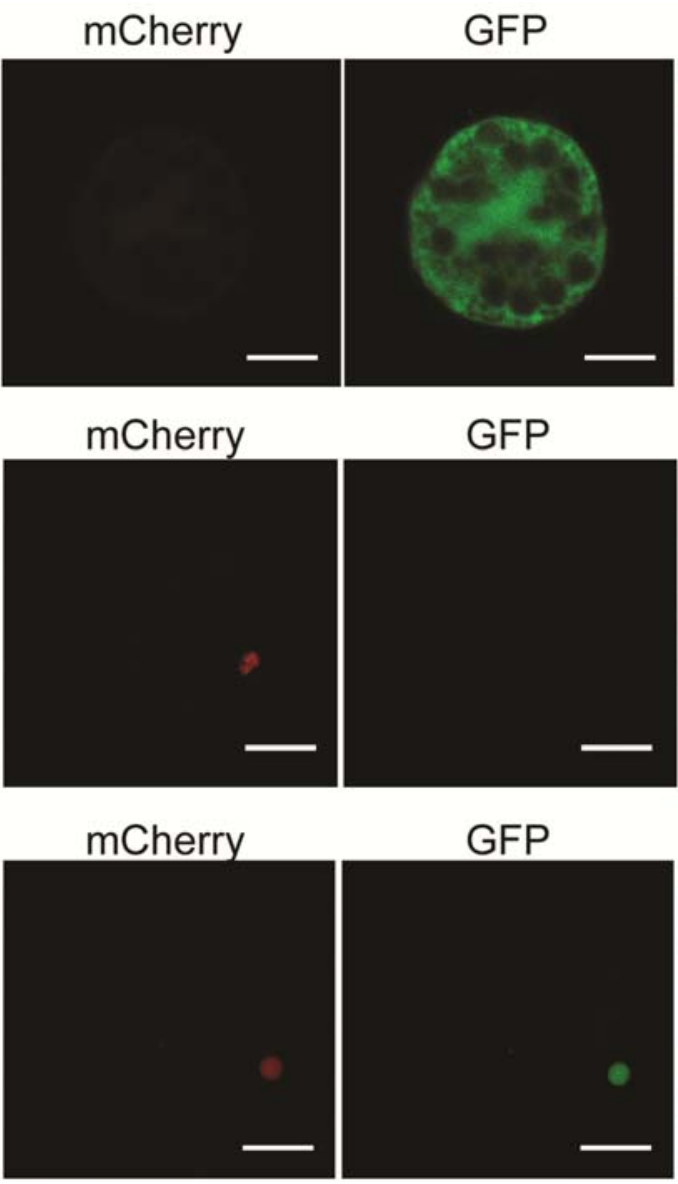

mCherry
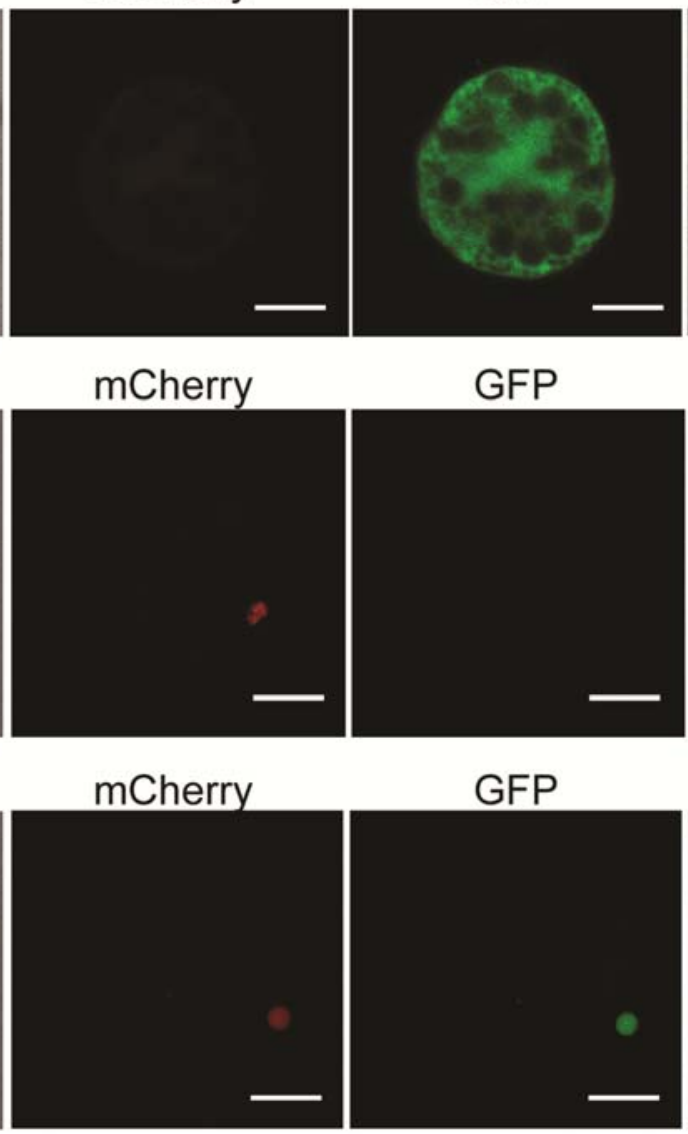

Merge

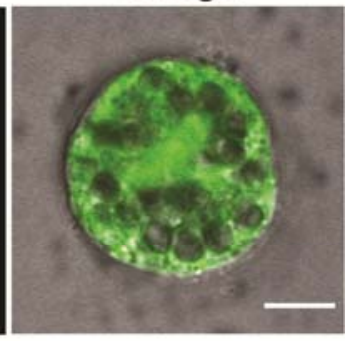

Merge

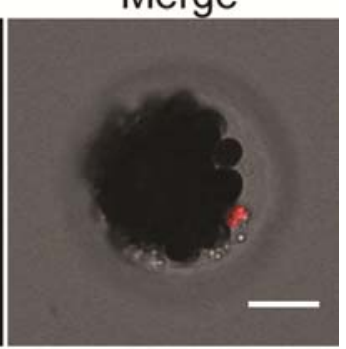

Merge

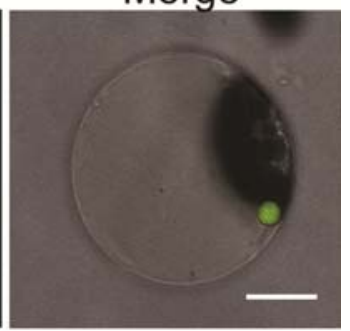

Fig. 6. Subcellular localization of the PhaTF15 protein. Green fluorescent protein (GFP), VirD2-NLS-mCherry (Lee et al. 2008), and PhaTF15-GFP fusion proteins were transiently expressed under control of the Cauliflower mosaic virus 35S promoter in protoplasts of Phalaenopsis aphrodite var. formosa. VirD2-NLS-mCherry was used as a nucleus marker to co-transfect protoplasts. Transfected protoplasts were incubated at $25^{\circ} \mathrm{C}$ in darkness for $16 \mathrm{~h}$, then examined under a confocal microscope. All PhaTF15-GFP and approximately $50 \%$ of GFP-PhaTF15 fusion protein localized in the nucleus of $P$. aphrodite var. formosa protoplasts and only PhaTF15-GFP is shown here. Scale bars represent $10 \mu \mathrm{m}$. 
sucrose at $5 \mathrm{~g}$ /liter, and $\mathrm{MgCl}_{2}$ at $0.5 \mathrm{~g} /$ liter) containing kanamycin at $100 \mathrm{mg} / \mathrm{liter}$ and $100 \mu \mathrm{M}$ acetosyringone at $28^{\circ} \mathrm{C}$ overnight. In total, $1 \mathrm{ml}$ of bacterial culture was then transferred into $10 \mathrm{ml}$ of YEB medium containing kanamycin at $100 \mathrm{mg} /$ liter and $100 \mu \mathrm{M}$ acetosyringone and further incubated at $28^{\circ} \mathrm{C}$ until the optical density reached approximately 1.0 to 1.2. A. tumefaciens cultures were centrifuged at $3,000 \times g$ for $10 \mathrm{~min}$, and cells were resuspended in $1 \mathrm{ml}$ of infiltration medium (10 mM morpholineethanesulfonic acid [MES], $10 \mathrm{mM}$ $\mathrm{MgCl}_{2}$, and $100 \mathrm{mM}$ acetosyringone) and incubated at room temperature for $3 \mathrm{~h}$. Two leaves of 1-year-old Phalaenopsis plants (six-leaf stage) were then infiltrated with $A$. tumefaciens suspension $(100 \mu \mathrm{l} /$ leaf $)$.

\section{RNA isolation and semiquantitative RT-PCR analysis.}

Total RNA was extracted from orchid plants as described (Tian et al. 1996). In total, $1 \mu \mathrm{g}$ of RNA was treated with RNase-free DNase (Ambion, Austin, TX, U.S.A.) for $0.5 \mathrm{~h}$ to eliminate genomic DNA contamination. Then, $0.5 \mu \mathrm{g}$ of DNAfree RNA was used for synthesis of first-strand cDNA by use of the Moloney murine leukemia virus reverse-transcriptase method (Invitrogen). PCR amplification involved gene-specific oligonucleotide primers, as described (Lu et al. 2007). Primer pairs were for CymMV, PhaPR1, PhaNPR1, and Ubiquitin 10 used as an internal control (Table 1). PCR products were separated by electrophoresis and visualized by ethidium bromide staining.

\section{Phylogenetic analysis.}

Phylogenetic analysis of PhaTF15 (accession number CB032966) and its homolog genes Zea mays (ACR37583), Arabi- dopsis thaliana (NP_564078), Brassica napa (ADK63399), C. annuum (ACN63363), Oryza sativa (NP_001050892), Picea sitchensis (ABR17325), Sorghum bicolor (XP_002442611), Ricinus communis (XP_002533271), and Glycine max (ACU19464) involved use of PAUP* 4.0b10 (Swofford 2002) by the neighbor-joining method. Bootstrap analysis was tested with 1,000 replicates and unweighted characters.

\section{Protoplast isolation and polyethylene glycol transfection.}

Protoplast isolation was as described (Yoo et al. 2007), with some modification. Tissue-cultured seedlings of $P$. aphrodite var. formosa purchased from the Taiwan Sugar Research Institute were used. In total, 1 to $5 \mathrm{~cm}$ of leaves were cut into 0.5 to 1-mm strips with use of a clean razor. The leaves were incubated in a petri dish with enzyme solution containing $1 \%$ cellulose R10 (Yakult Honsha, Tokyo), 0.2\% macerozyme R10 (Yakult Honsha), $0.4 \mathrm{M}$ mannitol, $20 \mathrm{mM} \mathrm{KCl,} 20 \mathrm{mM}$ MES, $10 \mathrm{mM} \mathrm{CaCl}_{2}$, and $0.1 \%$ bovine serum albumin, $\mathrm{pH} 5.7$, and incubated for $16 \mathrm{~h}$ in the dark. The protoplasts were harvested by spinning the enzyme solution at $100 \times g$ to pellet the protoplasts, then washed twice with W5 solution $(154 \mathrm{mM} \mathrm{NaCl}$, $125 \mathrm{mM} \mathrm{CaCl}_{2}, 5 \mathrm{mM} \mathrm{KCl}$, and $2 \mathrm{mM}$ MES, $\mathrm{pH}$ adjusted to 5.7), pelleted, and resuspended in $\mathrm{MMg}$ solution (0.4 M mannitol, $15 \mathrm{mM} \mathrm{MgCl}_{2}$, and $4 \mathrm{mM}$ MES, pH 5.7). Protoplasts were transfected by use of the polyethylene glycol (PEG) method (Yoo et al. 2007), with some modifications. Protoplasts $\left(1.5 \times 10^{5}\right.$ cells $)$ in $300 \mathrm{ml}$ of $\mathrm{MMg}$ solution were collected in a round-bottomed tube. Then, $50 \mu \mathrm{g}$ of each plasmid DNA and $330 \mu \mathrm{l}$ of PEG/Ca solution ( $4 \mathrm{~g}$ of PEG 4000, $3 \mathrm{ml}$ of $\mathrm{H}_{2} \mathrm{O}, 2.5$ $\mathrm{ml}$ of $0.8 \mathrm{M}$ mannitol, and $1 \mathrm{mM} \mathrm{CaCl}{ }_{2}$ ) was added to the tube smoothly for incubation at $23^{\circ} \mathrm{C}$ for $20 \mathrm{~min}$, and the tube was

Table 1. Primers used in the study

\begin{tabular}{|c|c|}
\hline Primer $^{\mathrm{a}}$ & Nucleotide sequence \\
\hline CymMV F1 & 5'-GAAAACCAAACCTCACGTCTAATCCGCTATCC-3' \\
\hline CymMV R1846 & 5'-TCCGGAAAGGGGCACTGGGGAACTGT-3' \\
\hline attP1 & 5'-CCAACTTTGTACAAAAAAGCTGAAC-3' \\
\hline attP2 & $5^{\prime}$-CCAACTTTGTACAAGAAAGCTGAAC-3' \\
\hline TF-attB1-F & 5'-GGGGACAAGTTTGTACAAAAAAGCAGGCTGCTCTAGAACTAGTGGATCCCCCG-3' \\
\hline TF-attB2-R ${ }^{b}$ & 5'-GGGGACCACTTTGTACAAGAAAGCTGGGTGCGAATTGGGTACCGGGCCCCCCC-3' \\
\hline PhaTF15 ORFF & 5'-ATGGCTGGGGGAGTTGGGGA-3' \\
\hline PhaTF15 ORFR & 5'-AAACCAAACAAGAGAAGCAATCCCAAGAGC-3' \\
\hline PhaTF15-hpRNA1 F & 5'-GGGGACAAGTTTGTACAAAAAAGCAGGCTCTATCCTCATTCATTTCATGG-3' \\
\hline PhaTF15-hpRNA1 R & 5'-GGGGACCACTTTGTACAAGAAAGCTGGGTCCATGAAATGAATGAGGATAG-3' \\
\hline PhaTF15-hpRNA2 F & 5'-GGGGACAAGTTTGTACAAAAAAGCAGGCTTGTTTCCATTGCTCAATTTCC-3' \\
\hline PhaTF15-hpRNA2 R & 5'-GGGGACCACTTTGTACAAGAAAGCTGGGTGGAAATTGAGCAATGGAAACA-3' \\
\hline PhaTF15-21IR R & 5'-GGGGACCACTTTGTACAAGAAAGCTGGGTCTATCCTCATTCATTTCATGG-3' \\
\hline CymMV CPF & 5'-GAAATAATCATGGGAGAGCC-3' \\
\hline CymMV CPR & 5'-AGTTTGGCGTTATT CAGTAGG-3' \\
\hline PR1 F & $5^{\prime}$-AGGACCCTGGCGTCTAAAG-3' \\
\hline PR1 R & 5'-TATTACAAATCAAACCGCTAAAG-3' \\
\hline NPR1 F & 5'-CGCATTTGTGTCGTAGTTTAT-3' \\
\hline NPR1 R & 5'-GGCCTTGCTCCTTTAGTTA-3' \\
\hline TF15 F & $5^{\prime}$-TGAATTAGCACAAGATCCGAT-3' \\
\hline TF15 R & 5'-AGCAATGGAAACAGACCACCAAT-3' \\
\hline Ubiquitin10 F & 5'-CCGGATCAGCAAAGGTTGA-3' \\
\hline Ubiquitin10 R & 5'-TCAGGCGGAGGACAAGATG-3' \\
\hline CymMV qPCR F & $5^{\prime}$-TGATGCTGGCCACTAACGATC-3' \\
\hline CymMV qPCR R & 5'-GGAATCAACGGCATCGAAGA-3' \\
\hline PR1 qPCR F & 5'-GGATCATCGTCTTGCGATTT-3' \\
\hline PR1 qPCR R & 5'-CCGCACAACTGTTACATGCAT-3' \\
\hline NPR1 qPCR F & 5'-AGTTGATGGCACCTTGGAGTTT-3' \\
\hline NPR1 qPCR R & 5'-AAGAAGCGTTTCCCGAGTTCA-3' \\
\hline TF15 qPCR F & 5'-GATCCACGCTCTAAATCCAATCC-3' \\
\hline TF15 qPCR R & 5'-AAATCTCGCACTGCCCAAAG-3' \\
\hline Ubiquitin10 qPCR F & $5^{\prime}$-CCGGATCAGCAAAGGTTGA-3' \\
\hline Ubiquitin10 qPCR R & 5'-AAGATTTGCATCCCTCCCC-3' \\
\hline
\end{tabular}

${ }^{a}$ CymMV = Cymbidium mosaic virus, $\mathrm{TF}=$ transcription factor, $\mathrm{ORF}=$ open reading frame, $\mathrm{PR}=$ pathogenesis related, and $\mathrm{qPCR}=$ quantitative polymerase chain reaction.

${ }^{\mathrm{b}}$ Underlined sequences indicated the attB sequences. The $4 \mathrm{G}$ at the most $5^{\prime}$ end were added as recommended by the manufacturer to increase the recombination rate. 
diluted with $1.32 \mathrm{ml}$ of W5 solution. The solution was gently mixed and centrifuged for $1 \mathrm{~min}$ to remove PEG. The protoplasts were resuspended in $5 \mathrm{ml}$ of $\mathrm{W} 5$ solution and incubated at $25^{\circ} \mathrm{C}$ in darkness.

\section{Confocal microscopy.}

Protoplasts used for observation of subcellular localization were examined by confocal microscopy (Leica TCS SP5 II) at the Joint Center for Instruments and Research, College of Bioresources and Agriculture, National Taiwan University. Protoplasts were harvested at $16 \mathrm{~h}$ post inoculation by centrifugation at $100 \times g$ for $5 \mathrm{~min}$. For simultaneous imaging of GFP and mCherry, GFP or mCherry fluorescence was excited by the 488- or 543-nm laser line, respectively, and detection spectrum range was from 500 to 587 or 600 to $630 \mathrm{~nm}$, respectively.

\section{LITERATURE CITED}

Bartlett, D. W., and Davis, M. E. 2006. Insights into the kinetics of siRNAmediated gene silencing from live-cell and live-animal bioluminescent imaging. Nucleic Acids Res. 34:322-333.

Dong, Y., Burch-smith, T. M., Liu, Y., Mamillapalli, P., and Dinesh-Kumar, S. P. 2007. A ligation-independent cloning tobacco rattle virus vector for high-throughput virus-induced gene silencing identifies roles for NbMADS4-1 and -2 in Floral development. Plant Physiol. 145:11611170.

Dressler, R. L. 1993. Phylogeny and Classification of the Orchid Family. Dioscorides Press, Portland, OR, U.S.A.

Durrant, W. E., and Dong, X. 2004. Systemic acquired resistance. Annu. Rev. Phytopathol. 42:185-209.

Grant, M., and Lamb, C. 2006. Systemic immunity. Curr. Opin. Plant Biol. 9:414-420.

Hartley, J. L., Temple, G. F., and Brasch, M. A. 2000. DNA Cloning using in vitro site-specific recombination. Genome Res. 10:1788-1795.

Karimi, M., Inze, D., and Depicker, A. 2002. GATEWAY vectors for Agrobacterium-mediated plant transformation. Trends Plant Sci. 7:193-195.

Kuttenkeuler, D., and Boutros, M. 2004. Genome-wide RNAi as a route to gene function in Drosophila. Brief. Funct. Genomics Proteomics 3:168176.

Lee, L. Y., Fang, M. J., Kuang, L. Y., and Gelvin, S. B. 2008. Vectors for multi-color bimolecular fluorescence complementation to investigate protein-protein interactions in living plant cells. Plant Methods 4:24.

Liu, Y., Schiff, M., and Dinesh-Kumar, S. P. 2002. Virus-induced gene silencing in tomato. Plant J. 31:777-786.

Loake, G., and Grant, M. 2007. Salicylic acid in plant defence-the players and protagonists. Curr. Opin. Plant Biol. 10:466-472.

Lu, H. C., Chen, H. H., Tsai, W. C., Chen, W. H., Su, H. J., Chang, C. N., and Yeh, H. H. 2007. Strategies for functional validation of genes involved in reproductive stages of orchids. Plant Physiol. 143:558-569.

Lu, R., Malcuit, I., Moffett, P., Ruiz, M. T., Peart, J., Wu, A. J., Rathjen, J. P., Bendahmane, A., Day, L., and Baulcombe, D. C. 2003. High throughput virus-induced gene silencing implicates heat shock protein 90 in plant disease resistance. EMBO (Eur. Mol. Biol. Organ.) J. 22:5690-5699.

Ma, K., Xiao, J., Li, X., Zhang, Q., and Lian, X. 2009. Sequence and expression analysis of the C3HC4-type RING finger gene family in rice. Gene 444:33-45.

Martin, S. E., and Caplen, N. J. 2007. Applications of RNA interference in mammalian systems. Annu. Rev. Genomics Hum. Genet. 8:81-108.
McDowell, J. M., and Dangl, J .L. 2000. Signal transduction in the plant immune response. Trends Biochem. Sci. 25:79-82.

Mou, Z., Fan, W., and Dong, X. 2003. Inducers of plant systemic acquired resistance regulate NPR1 function through redox changes. Cell 113:935-944.

Ohkumo, T., Masutani, C., Eki, T., and Hanaoke, F. 2008. Use of RNAi in C. elegans. Methods Mol. Biol. 442:129-137.

Pieterse, C. M. J., and Van Loon, L. C. 2004. NPR1: the spider in the web of induced resistance signaling pathways. Curr. Opin. Plant Biol. 7:456464.

Robertson, D. 2004. VIGS vectors for gene silencing: many targets, many tools. Annu. Rev. Plant Biol. 55:495-519.

Shang, Y., Schwinn, K. E., Bennett, M. J., Hunter, D. A., Waugh, T. L. Pathirana, N. N., Brummell, D. A., Jameson, P. E., and Davies, K. M. 2007. Methods for transient assay of gene function in floral tissues. Plant Methods 3:1-12.

Small, G. W., Shi, Y. Y., Higgins, L. S., and Orlowski, R. Z. 2007.Mitogenactivated protein kinase phosphatase-1 is a mediator of breast cancer chemoresistance. Cancer Res. 67:4459-4466.

Sticher, L., Mauch-Mani, B., and Metraux, J. P. 1997. Systemic acquired resistance. Annu. Rev. Phytopathol. 35:235-270.

Swofford, D. L. 2002. PAUP*: Phylogenetic Analysis Using Parsimony (*and other methods), version 4.0beta10. Sinauer Associates, Inc., Sunderland, MA, U.S.A.

Tian, T., Klaassen, V. A., Soong, J., Wisler, G., Duffus, J. E., and Falk, B. W. 1996. Generation of cDNAs specific to Lettuce infectious yellows closterovirus and other whitefly-transmitted viruses by RT-PCR and degenerate oligonucleotide primers corresponding to the closterovirus gene encoding the heat shock protein 70 homolog. Phytopathology 86:1167-1173

Tsai, W. C., Hsiao, Y. Y., Lee, S. H., Tung, C. W., Wang, D. P., Wang, H. C., Chen, W. H., and Chen, H. H. 2006. Expression analysis of the ESTs derived from the flower buds of Phalaenopsis equestris. Plant Sci. 107:426-43.

Uknes, S., Mauch-Mani, B., Moyer, M., Potter, S., Williams, S., Dincher, S., Chandler, D., Slusarenko, A., Ward, E., and Ryals, J. 1992. Acquired resistance in Arabidopsis. Plant Cell 4:645-656.

Van Loon, L. C., and Van Kammen, A. 1970. Polyacrylamide disc electrophoresis of the soluble leaf protein from Nicotiana tabacum var. 'Samsun' and 'Samsun NN'. II. Changes in protein constitution after infection with tobacco mosaic virus. Virology 40:199-211.

Van Loon, L. C., and Van Strien, E. A. 1999. The families of pathogenesisrelated proteins, their activities, and comparative analysis of PR-1 type proteins. Physiol. Mol. Plant Pathol. 55:85-97.

Vlot, A. C., Dempsey, D. A., and Klessig, D. F. 2009. Salicylic acid, a multifaceted hormone to combat disease. Annu. Rev. Phytopathol. 47:177-206.

Wang, M. B., and Waterhouse, P. M. 2002. Application of gene silencing in plants. Curr. Opin. Plant Biol. 5:146-150.

Watson, J. M., Fusaro, A. F., Wang, M., and Waterhouse, P. M. 2005. RNA silencing platforms in plant. FEBS (Fed. Eur. Biochem. Soc.) Lett. 579:5982-5987.

Yoo, S. D., Cho, Y. H., and Sheen, J. 2007. Arabidopsis mesophyll protoplasts: a versatile cell system for transient gene expression analysis. Nat. Protoc. 2:1565-1572.

Yu, D., Chen, C., and Chen, Z. 2001. Evidence for an important role of WRKY DNA binding proteins in the regulation of NPRl gene expression. Plant Cell 13:1527-1539.

Zeba, N., Isbat, M., Kwon, N. J., Lee, M. O., Kim, S. R., and Hong, C. B. 2009. Heat-inducible C3HC4 type RING zinc finger protein gene from Capsicum annuum enhances growth of transgenic tobacco. Planta 229:861-871.

\section{ACKNOWLEDGMENTS}

This research was funded through grants from the National Science Council, Taiwan (grant nos. 98-2321-B-002-019-MY3, 98-2313- B-002-043-MY3).

Added to the online article on May 31, 2012. 